CLINICAL STUDY

\title{
Effects of rosiglitazone on fasting plasma fibroblast growth factor-21 levels in patients with type 2 diabetes mellitus
}

\author{
$\mathrm{Ke} \mathrm{Li}^{*}$, Ling Li ${ }^{1, *}$, Mengliu Yang, Haihong Zong ${ }^{2}$, Hua Liu ${ }^{3}$ and Gangyi Yang \\ Department of Endocrinology, the Second Affiliated Hospital, Chongqing Medical University, 400010 Chongqing, People's Republic of China, ${ }^{1}$ The Key \\ Laboratory of Laboratory Medical Diagnostics in the Ministry of Education and Department of Clinical Biochemistry, Chongqing Medical University, \\ 400016 Chongqing, People's Republic of China, ${ }^{2}$ Department of Medicine/Endocrinology, Albert Einstein College of Medicine, 1301 Morris Park Avenue, \\ Bronx, New York 10461, USA and ${ }^{3}$ Department of Pediatrics, University of Mississippi Medical Center, 2500 North State Street, Jackson, Mississippi \\ 39216-4505, USA \\ (Correspondence should be addressed to G Y Yang; Email: gangyiyang@yahoo.com.cn)
}

*(L Li and K Li contributed equally to this work)

\begin{abstract}
Objective: Fibroblast growth factor-21 (FGF-21) has recently been characterized as a potent metabolic regulator, but its pathophysiologic roles in humans remain unknown. This study aimed to investigate the effects of rosiglitazone on plasma FGF-21 levels in patients with type 2 diabetes mellitus (T2DM). Design and methods: Thirty patients with new-onset T2DM (nT2DM), 34 type 2 diabetic patients with poor glycemic control (pT2DM) after the treatment with single hypoglycemic agent metformin, and 30 sex- and age-matched normal glycaemic controls (NGT) participated in the study. The pT2DM group was treated with rosiglitazone for 12 weeks. Plasma FGF-21 levels were measured with a RIA. The relationship between plasma FGF-21 levels and metabolic parameters was also analyzed. Results: Fasting plasma FGF-21 levels were higher in nT2DM and pT2DM groups than in the control $(1.81 \pm 0.64$ vs $1.87 \pm 0.63$ vs $1.52 \pm 0.61 \mu \mathrm{g} / \mathrm{l}, P<0.05)$, but there was no difference between nT2DM and pT2DM groups. Fasting plasma FGF-21 levels were decreased significantly in pT2DM group after the treatment with rosiglitazone compared with pre-treatment $(1.59 \pm 0.63$ vs $1.87 \pm 0.64 \mu / 1, P<0.05)$. In all diabetic patients, multiple regression analysis showed that HbA1c, fasting insulin, and homeostasis model assessment-insulin resistance index were independently associated with plasma FGF-21 levels.

Conclusions: In pT2DM patients, plasma FGF-21 levels are increased, but significantly decreased after the treatment with rosiglitazone on top of ongoing metformin therapy. These data suggest that rosiglitazone may play a role in lowering FGF-21 levels in T2DM patients.
\end{abstract}

European Journal of Endocrinology 161 391-395

\section{Introduction}

Impaired insulin-stimulated glucose turnover in insulindependent peripheral tissues such as liver, muscle, and adipocytes is a major characteristic of type 2 diabetes. Fibroblast growth factor-21 (FGF-21) is a liver-derived endocrine factor that influences insulin sensitivity in adipocytes and whole-body glucose homeostasis in animal models (1). A recent report has shown that FGF-21 is a potent activator of glucose transport in mouse adipocytes, and its glucose-lowering effect was through an insulin-independent mechanism (2). Mice with over-expression of FGF-21 were protected from diet-induced obesity (2). Zhang et al. observed that serum FGF-21 levels in overweight/obese Chinese subjects were higher than in lean Chinese individuals, and that the FGF-21 levels correlated positively with adiposity as well as fasting insulin (FINS) and triglycerides (TGs) (3). The authors speculated that the increased serum FGF-21 levels in obese individuals could be a result of compensatory responses or resistance to FGF21 actions. The anti-diabetic effects of FGF-21 and its regulatory mechanism in humans remain unknown despite the research efforts done in animals.

Rosiglitazone, an agonist of the peroxisome proliferators-activated receptor- $\gamma(\operatorname{PPAR}-\gamma)$, regulates the expression of genes controlling glucose homeostasis and lipid metabolism. Rosiglitazone has been widely used as a pharmacological agent in clinic to alleviate peripheral insulin resistance (IR) and enhance glucose transport in adipose tissue $(4,5)$. Previous studies have suggested a functional interplay between FGF-21 and PPAR- $\gamma$-signaling pathways $(6,7)$. Muise et al. reported that circulating levels of FGF21 protein were increased in $\mathrm{db} / \mathrm{db}$ mice treated with the PPAR- $\gamma$ agonists (8).

Since the role of FGF21 in human physiology is unknown, it is important to characterize the effect of 
common anti-diabetic treatment on the plasma levels of FGF21. Since monotherapy with rosiglitazone has limited effect on plasma glucose, the metformin was combined to control the high plasma glucose level. We present here a cross-sectional study of FGF-21 levels in different populations as well as the effects of rosiglitazone on the plasma FGF-21 levels in patients with type 2 diabetes.

\section{Patients and methods}

\section{Subjects}

Ninety-four Chinese volunteers were involved in this study and categorized into three groups. The first group comprised of 30 otherwise healthy individuals except the diagnosis of new-onset type 2 diabetes mellitus (nT2DM). The diagnostic criteria of type 2 diabetes were based on a $75 \mathrm{~g}$ oral glucose tolerance test (OGTT) recommended by World Health Organization criteria (9). These patients had not undertaken any diabetic medications/diet prior to the present study.

The second group of 34 patients was type 2 diabetes patients with poor glycemic control (pT2DM) even after the treatment with metformin. The selection criteria included: 1) 40-70 years of age without the presence of major diabetic complications and major organ diseases; 2) fasting blood glucose levels between 7.0 and $13.9 \mathrm{mmol} / \mathrm{l}$ after more than 3 months of treatment with metformin; and 3) body mass index (BMI) within the range of $20-30 \mathrm{~kg} / \mathrm{m}^{2}$.

Thirty healthy volunteers who responded to our advertisement and age- and BMI-matched with the diabetic group, were chosen as the normal glycemic control group (NGT). Their non-diabetic statuses were confirmed with a normal OGTT.

This study was carried out in accordance with the recommendations of the Declaration of Helsinki. The study was approved by the Human Research Ethics Committee of Chongqing Medical University. An informed consent was obtained from all participants in this study.

\section{Study design}

Rosiglitazone (4 mg/day) was added to the previous metformin regimen in patients of pT2DM group throughout the 12-week period of study. No change in medication and dosages was made during the study. No subject dropped out of the study. The NGT control and the nT2DM group did not receive any medication.

\section{Plasma biochemical parameters and FGF-21}

Blood samples were taken before and after the 12 weeks of treatment for the measurements of metabolic parameters and plasma FGF-21 levels. Typically, blood samples were collected either after an overnight fast or $2 \mathrm{~h}$ after a $75 \mathrm{~g}$ OGTT. Plasma samples were collected by centrifugation at $4{ }^{\circ} \mathrm{C}$ and kept at $-80{ }^{\circ} \mathrm{C}$ for further use.

Plasma FGF-21 levels were determined by RIA (Phoenix Pharmaceuticals, Inc., Belmont, CA, USA) using ${ }^{125}$ I-labeled FGF-21 as tracer. The linear range of the assay was $0.5-5.0 \mu \mathrm{g} / \mathrm{l}$, and the standard range was $0.234-30 \mu \mathrm{g} / \mathrm{l}$. The inter- and intra-assay coefficients of variation were 4.9 and $13 \%$ respectively. Insulin was measured in deproteinized serum by RIA using human insulin as standards (Linco, St Charles, MO, USA). Free fatty acids (FFA) were measured with a commercial assay kit (Randox Laboratories Ltd, Antrim, UK). Plasma glucose was assayed using the glucose oxidase method. HbA1c was measured by isoelectric focusing. TG, cholesterol, high-density lipoprotein (HDL), and low-density lipoprotein (LDL) concentrations were determined enzymatically. Percent body fat was determined by bioelectrical impedance analysis (Tanita, Inc., Tokyo, Japan). The homeostasis model assessment of IR (HOMA-IR) and the HOMA of $\beta$-cell insulin secretion (HOMA-IS) were calculated from FINS and glucose levels using the following equations:

$$
\begin{aligned}
\mathrm{HOMA}-\mathrm{IR}= & \text { fasting insulin }(\mathrm{mU} / \mathrm{l}) \\
& \times \text { fasting blood glucose }(\mathrm{mmol} / \mathrm{l}) / 22.5 ;
\end{aligned}
$$

HOMA - IS $=(20 \times$ fasting insulin $(\mathrm{mU} / \mathrm{l})) /($ fasting $\times$ blood glucose $(\mathrm{mmol} / \mathrm{l})-3.5)$

(Matthews et al. 1985).

\section{Statistical analysis}

Statistical analyses were performed using the SAS 8.0 software (SAS Inc., Cary, NC, USA). Since the distributions of plasma insulin, TG, HOMA-IR, and HOMR-IS values were skewed, logarithmically transformed values were used for statistical analysis. Baseline characteristics of case and control subjects were compared by $t$-test or ANOVA. The paired $t$-test was used to compare differences in biochemical characteristics and FGF-21 levels between pre- and post-treatment with rosiglitazone in pT2DM group. One-way ANOVA and Tukey's honestly significant difference post hoc test were performed to test the changes between the groups. Bivariate correlation and multiple regression analyses were used to examine the association between fasting plasma FGF-21 levels and the values of other biomarkers. All of the statistical analyses were twosided, and all data are presented as means \pm s.D. or medians (interquartile ranges) with a $P$ value $<0.05$ considered statistically significant. 


\section{Results}

\section{The clinical characteristics and fasting plasma FGF-21 levels}

The clinical characteristics of the three groups did not show significant difference in gender distribution, age, BMI, waist-to-hip ratio (WHR), percent body fat, systolic, and diastolic blood pressure (Table 1). The fasting levels of TG, HDL, LDL, FFA, and post-prandial insulin (PINS) are comparable between the groups. The pT2DM group has higher total cholesterol (TC) than the other two groups $(P<0.05)$. Both pT2DM and nT2DM groups had significantly higher fasting blood glucose, 2-h post-prandial blood glucose (2hPBG), FINS, HbA1c, HOMA-IR, and fasting plasma FGF-21 levels, but lower HOMA-IS than the NGT group. The fasting blood glucose, 2hPBG, and $\mathrm{HbA1c}$ in nT2DM group were significantly higher than in pT2DM group. There was no significant difference in FINS, HOMA-IR, HOMA-IS, and fasting plasma FGF-21 levels between the pT2DM and nT2DM groups.

\section{Relationship between fasting plasma FGF-21 levels and metabolic parameters}

In NGT group, fasting plasma FGF-21 levels correlated positively with BMI $(r=0.39, P=0.031)$ in simple regression analysis, while in multiple stepwise regression analysis, BMI was independently related to plasma FGF-21 levels. The multiple regression equation was: $Y_{\mathrm{FGF}-21}=-0.602+0.088 \mathrm{X}_{\mathrm{BMI}}$. In all diabetes patients, gender, age, BMI, WHR, TG, TC, LDL, HDL-C, FFA, FINS, HbA1c, BP, FINS, PINS, HOMA-IR, and HOMA-IS were included in the multiple regression. Multiple regression analyses with backward exclusion showed that HbA1c, FINS, and HOMA-IR were independently related to fasting plasma FGF-21 levels. The multiple regression equation was

$$
\begin{aligned}
\mathrm{Y}_{\mathrm{FGF}-21}= & 3.012-0.242 \mathrm{X}_{\mathrm{HbAlc}}-0.101 \mathrm{X}_{\mathrm{FINS}} \\
& +1.273 \mathrm{X}_{\mathrm{HOMA}-\mathrm{IR}} .
\end{aligned}
$$

\section{The effects of rosiglitazone on clinical characteristics and FGF-21 levels in PT2DM group}

To examine the effect of rosiglitazone on type 2 diabetes, we added rosiglitazone to the metformin regimen in pT2DM group. After 12 weeks treatment with rosiglitazone and metformin, HbA1c, FBG, 2hPBG, FINS, and HOMA-IR in pT2DM patients were significantly declined (Table 2). Interestingly, fasting plasma FGF-21 levels were also significantly decreased $(1.59 \pm 0.63$ vs $1.87 \pm 0.64 \mu \mathrm{g} / \mathrm{l}, P<0.05)$. There was no significant change in BMI, percent body fat, and HOMA-IS, while

\begin{tabular}{|c|c|c|c|}
\hline Group & pT2DM & nT2DM & NGT \\
\hline Gender (M:F) & $11: 23$ & $12: 18$ & $11: 19$ \\
\hline Age (years) & $57.26 \pm 8.56$ & $58.80 \pm 6.80$ & $57.93 \pm 6.46$ \\
\hline Disease duration (years) & $4.6 \pm 0.30$ & - & - \\
\hline BMI $\left(\mathrm{kg} / \mathrm{m}^{2}\right)$ & $25.15 \pm 2.95$ & $24.18 \pm 2.78$ & $23.94 \pm 2.55$ \\
\hline WHR & $0.87+0.05$ & $0.89+0.08$ & $0.87+0.05$ \\
\hline Body fat (\%) & $32.48 \pm 6.62$ & $31.93 \pm 7.89$ & $28.60 \pm 7.54$ \\
\hline $\mathrm{SBP}(\mathrm{mmHq})$ & $124.77+10.95$ & $125.63+19.76$ & $117.47+9.35$ \\
\hline $\mathrm{DBP}(\mathrm{mmHg})$ & $75.21 \pm 6.79$ & $78.10 \pm 10.34$ & $73.60 \pm 6.58$ \\
\hline $\mathrm{TG}(\mathrm{mmol} / \mathrm{l})$ & $1.69(0.67-5.81)$ & $1.33(0.45-4.03)$ & $1.07(0.60-4.11)$ \\
\hline $\mathrm{TC}(\mathrm{mmol} / \mathrm{l})$ & $5.12 \pm 0.87^{\star \dagger}$ & $4.66 \pm 0.80$ & $4.21 \pm 1.06$ \\
\hline $\mathrm{HDL}(\mathrm{mmol} / \mathrm{l})$ & $1.30 \pm 0.32$ & $1.24 \pm 0.27$ & $1.21 \pm 0.42$ \\
\hline $\mathrm{LDL}(\mathrm{mmol} / \mathrm{l})$ & $2.88+0.73$ & $2.66+0.63$ & $2.52+0.77$ \\
\hline $\mathrm{FFA}(\mu \mathrm{mol} / \mathrm{l})$ & $0.69 \pm 0.27$ & $0.70 \pm 0.27$ & $0.59 \pm 0.18$ \\
\hline $\mathrm{HbA} 1 \mathrm{c}(\%)$ & $6.77+1.16^{\star \top}$ & $8.39+2.48^{\star}$ & $5.42+0.38$ \\
\hline $\mathrm{FBG}(\mathrm{mmol} / \mathrm{l})$ & $7.99 \pm 1.25^{\star \dagger}$ & $9.85 \pm 4.11^{*}$ & $5.36 \pm 0.47$ \\
\hline $2 \mathrm{hPBG}(\mathrm{mmol} / \mathrm{l})$ & $14.02 \pm 3.75^{\star \dagger}$ & $18.51 \pm 7.81^{*}$ & $6.02 \pm 0.91$ \\
\hline FINS (mU/l) & $10.77(5.84-37.80)^{\star}$ & $8.91(6.17-29.45)^{*}$ & $9.89(3.07-24.74)$ \\
\hline PINS (mU/l) & $36.13(11.26-122.28)$ & $28.16(8.77-98.88)$ & $35.01(16.13-86.20)$ \\
\hline HOMA-IR & $4.08(1.77-13.67)^{*}$ & $4.38(2.14-13.28)^{*}$ & $2.26(0.79-6.03)$ \\
\hline HOMA-IS & $50.54(22.82-162.92)^{\star}$ & $42.46(11.07-236.57)^{\star}$ & $112.64(26.71-249.90)$ \\
\hline FGF-21 ( $\mu \mathrm{g} / \mathrm{l})$ & $1.87 \pm 0.64^{*}$ & $1.81 \pm 0.63^{*}$ & $1.52 \pm 0.61$ \\
\hline ACE inhibitors & 3 & 4 & 0 \\
\hline$\beta$-Blockers & 0 & 1 & 0 \\
\hline Diuretics & 1 & 0 & 0 \\
\hline Statins & 5 & 2 & 0 \\
\hline Fibrates & 1 & 0 & 0 \\
\hline
\end{tabular}

Table 1 Clinical characteristics and fibroblast growth factor-21 (FGF-21) levels of study subjects.

Data are means \pm S.D. or medians (interquartile ranges) versus NGT group ${ }^{\star} P<0.05$; T2DM group versus nT2DM group ${ }^{\dagger} P<0.05$. pT2DM, type 2 diabetes mellitus with poor glycemic control; nT2DM, new-onset type 2 diabetes mellitus; NGT, normal glycaemic control; BMI, body mass index; WHR, waist-to-hip ratio; SBP, systolic blood pressure; DBP, diastolic blood pressure; TG, total triglyceride; TC, total cholesterol; HDL, high-density lipoprotein; LDL, low-density lipoprotein; FFA, free fatty acids; FBG, fasting blood glucose; 2hPBG, 2-h post-prandial blood glucose; FINS, fasting insulin; PINS, 2-h post-prandial insulin; HOMA-IR, HOMA-insulin resistance index; HOMA-IS, HOMA- $\beta$-cell insulin secretion index; FGF-21, fibroblast growth factor-21. 
a remarkably reduced FFA level $(0.38 \pm 0.18$ vs $0.69 \pm$ $0.27 \mu \mathrm{mol} / \mathrm{l}, P<0.05)$ was observed after the addition of rosiglitazone in the pT2DM group (Table 2).

\section{Discussion}

In this study, we assessed association between fasting plasma FGF-21 and several metabolic characteristics in T2DM and non-diabetic controls. In NGT group, fasting plasma FGF-21 levels only correlated positively with BMI, whereas in T2DM group, HbA1c, FINS, and HOMA-IR were independently related to fasting plasma FGF-21 levels. These findings suggest a possible interaction between FGF-21 and insulin action/secretion in type 2 diabetes. However, we also observed no difference in FGF-21 levels between pT2DM and nT2DM groups, despite the nT2DM group having higher levels of plasma glucose. These results suggest that FGF-21 might play a role in mediating insulin secretion/actions and that insulin might be an important factor to FGF-21 level or action.

FGF21 has previously been shown to lower blood glucose, improving peripheral insulin sensitivity (10), enhancing glucose transportation in adipocytes, and preventing pancreatic $\beta$-cells from apoptosis (2). It is well known that GLUT-1 is responsible for basal glucose uptake in adipocytes, and insulin-stimulated glucose uptake is through the translocation of GLUT-4 from intracellular storage vesicles to the plasma

Table 2 Clinical characteristics and fibroblast growth factor-21 (FGF-21) levels pre- and post-treatment with rosiglitazone in pT2DM group.

\begin{tabular}{lll}
\hline Group & Pre-treatment & Post-treatment \\
\hline BMI $\left(\mathrm{kg} / \mathrm{m}^{2}\right)$ & $25.15 \pm 2.95$ & $25.43 \pm 2.93$ \\
WHR & $0.87 \pm 0.05$ & $0.87 \pm 0.06$ \\
Body fat $(\%)$ & $32.48 \pm 6.62$ & $33.04 \pm 6.68$ \\
SBP $(\mathrm{mmHg})$ & $124.77 \pm 10.95$ & $126.91 \pm 12.91$ \\
DBP $(\mathrm{mmHg})$ & $75.21 \pm 6.79$ & $76.50 \pm 8.61$ \\
TG $(\mathrm{mmol} / \mathrm{l})$ & $1.69(0.67-5.81)$ & $1.69(0.54-7.46)$ \\
TC $(\mathrm{mmol} / \mathrm{l})$ & $5.12 \pm 0.87$ & $5.10 \pm 1.12$ \\
HDL $(\mathrm{mmol} / \mathrm{l})$ & $1.30 \pm 0.32$ & $1.23 \pm 0.28$ \\
LDL $(\mathrm{mmol} / \mathrm{l})$ & $2.88 \pm 0.73$ & $2.95 \pm 0.72$ \\
FFA $(\mu \mathrm{mol} / \mathrm{l})$ & $0.69 \pm 0.27$ & $0.38 \pm 0.18^{*}$ \\
HbA1c $(\%)$ & $6.77 \pm 1.16$ & $6.32 \pm 0.57^{*}$ \\
FBG $(\mathrm{mmol} / \mathrm{l})$ & $7.99 \pm 1.25$ & $6.88 \pm 0.94^{*}$ \\
2hPBG $(\mathrm{mmol} / \mathrm{l})$ & $14.02 \pm 3.75$ & $9.64 \pm 2.57^{*}$ \\
FINS $(\mathrm{mU} / \mathrm{l})$ & $10.77(5.84-37.80)$ & $10.49(5.14-17.79)^{\star}$ \\
PINS $(\mathrm{mU} / \mathrm{l})$ & $36.13(11.26-122.28)$ & $33.28(10.70-76.84)$ \\
HOMA-IR & $4.08(1.77-13.67)$ & $2.99(1.54-5.39)^{*}$ \\
HOMA-IS & $50.54(22.82-162.92)$ & $58.39(30.58-128.04)$ \\
FGF-21 $(\mu \mathrm{g} / \mathrm{l})$ & $1.87 \pm 0.64$ & $1.59 \pm 0.63^{*}$ \\
\hline
\end{tabular}

Data are means \pm S.D. or medians (interquartile ranges) versus pre-treatment ${ }^{\star} P<0.05$. Pre-treatment, pre-treatment with rosiglitazone; post-treatment, post-treatment with rosiglitazone; BMI, body mass index; WHR, waist-to-hip ratio; SBP, systolic blood pressure; DBP, diastolic blood pressure; TG, total triglyceride; TC, total cholesterol; HDL, high-density lipoprotein; LDL, lowdensity lipoprotein; FFA, free fatty acids; FBG, fasting blood glucose; 2hPBG, 2-h post-prandial blood glucose; FINS, fasting insulin; PINS, 2-h postprandial insulin; HOMA-IR, HOMA-insulin resistance index; HOMA-IS, HOMA- $\beta$-cell insulin secretion index; FGF-21, fibroblast growth factor-21. membrane. The suppression of GLUT-4 expression and translocation in T2DM contribute to reduced whole-body glucose clearance and raised plasma glucose levels (11). However, the effects of FGF-21 on glucose transportation in adipose tissue were through insulin-independent upregulation of GLUT-1 expression (2). We have previously demonstrated that fasting FGF-21 levels were significantly increased in patients with T2DM, which may be explained by a compensatory response to defective insulin action (12). We also observed that plasma FGF-21 levels were higher in patients with diabetic ketosis, consistent with the results reported by Muise et al. in $\mathrm{db} / \mathrm{db}$ mice $(8,13)$. A study using ELISA assay, in contrast to the RIA method in our study, reported that serum FGF21 varied 250-fold among 76 healthy human individuals and that induction of ketogenesis was independent of FGF21 levels (14).

We observed a decrease in fasting plasma FGF-21 after treatment with rosiglitazone in pT2DM, which was discrepant to the results in $\mathrm{db} / \mathrm{db}$ mice reported by Muise et al. This discrepancy could be caused by the improved insulin sensitivity shown in our study. Wang et al. have identified an amino acid of the ligand-binding domain that is required for the response of PPAR- $\gamma$ to endogenous ligands, which is also required for expression of FGF-21 (6). It was previously reported that rosiglitazone enhanced FGF-21 action to induce tyrosine phosphorylation of FGF receptor (FGFR)-2 through activating the PPAR- $\gamma$ pathway in 3T3-L1 adipocytes (7). FGFR1c, FGFR3c, and $\beta$-klotho are thought to be determined in the action of FGF21 in several current studies $(15,16)$. Thus, rosiglitazone may improve FGF-21 action through the PPAR- $\gamma$ pathway, and consequently alleviate FGF-21 resistance, and reduce plasma FGF-21 levels, but this needs further investigation.

Patients in pT2DM group were on single hypoglycemic agent metformin before participating in the study of rosiglitazone. Thus, the effects of rosiglitazone on the plasma levels of glucose, insulin, and FGF-21 reported here need to be further clarified. The difference in plasma FGF-21 levels between our study and the study done by Gälman et al. is probably due to the different sensitivity of assay methods (ELISA versus RIA). The plasma FGF-21 levels measured by both methods should be evaluated in our future studies.

Our findings suggest that insulin might be an important factor for FGF-21 level, and FGF-21 pathway might be a compensatory mechanism for impaired insulin action. The administration of rosiglitazone on top of ongoing metformin therapy decreases FGF-21 levels, probably through lowering plasma glucose and improving insulin sensitivity. Thus, FGF-21 might be an attractive therapeutic agent for T2DM. The biological mechanism of FGF-21 action and its interaction with insulin in humans need further investigation. 


\section{Declaration of interest}

The authors have nothing to disclose.

\section{Funding}

This work was supported by research grants from the National Natural Science Foundation of China (30871199 and 30771037), Chongqing Municipal Education Commission (KJ050304), and Chongqing Medical University (XBZD200704).

\section{References}

1 Nishimura T, Nakatake Y, Konishi M \& Itoh N. Identification of a novel FGF, FGF-21, preferentially expressed in the liver. Biochimica et Biophysica Acta 20001492 203-206.

2 Kharitonenkov A, Shiyanova TL, Koester A, Ford AM, Micanovic R, Galbreath EJ, Sandusky GE, Hammond LJ, Moyers JS, Owens RA, Gromada J, Brozinick JT, Hawkins ED, Wroblewski VJ, Li DS, Mehrbod F, Jaskunas SR \& Shanafelt AB. FGF-21 as a novel metabolic regulator. Journal of Clinical Investigation 2005115 1627-1635.

3 Zhang X, Yeung DC, Karpisek M, Stejskal D, Zhou ZG, Liu F, Wong RL, Chow WS, Tso AW, Lam KS \& Xu A. Serum FGF21 levels are increased in obesity and are independently associated with the metabolic syndrome in humans. Diabetes 200857 1246-1253.

4 Mayerson AB, Hundal RS, Dufour S, Lebon V, Befroy D, Cline GW, Enocksson S, Inzucchi SE, Shulman GI \& Petersen KF. The effects of rosiglitazone on insulin sensitivity, lipolysis, and hepatic and skeletal muscle triglyceride content in patients with type 2 diabetes. Diabetes 200251 797-802.

5 Ciaraldi TP, Kong AP, Chu NV, Kim DD, Baxi S, Loviscach M, Plodkowski R, Reitz R, Caulfield M, Mudaliar S \& Henry RR. Regulation of glucose transport and insulin signaling by troglitazone or metformin in adipose tissue of type 2 diabetic subjects. Diabetes 200251 30-36.

6 Wang H, Qiang L \& Farmer SR. Identification of a domain within peroxisome proliferator-activated receptor gamma regulating expression of a group of genes containing fibroblast growth factor 21 that are selectively repressed by SIRT1 in adipocytes. Molecular and Cellular Biology 200828 188-200.

7 Moyers JS, Shiyanova TL, Mehrbod F, Dunbar JD, Noblitt TW, Otto KA, Reifel-Miller A \& Kharitonenkov A. Molecular determinants of FGF-21 activity-synergy and cross-talk with PPAR $\gamma$ signaling. Journal of Cellular Physiology 2007210 1-6.
8 Muise ES, Azzolina B, Kuo DW, El-Sherbeini M, Tan Y, Yuan X, $\mathrm{Mu}$ J, Thompson JR, Berger JP \& Wong KK. Adipose fibroblast growth factor 21 is up-regulated by peroxisome proliferatoractivated receptor gamma and altered metabolic states. Molecular Pharmacology 2008 74 403-412.

9 Alberti KG \& Zimmet PZ. Definition, diagnosis and classification of diabetes mellitus and its complications. Part 1: diagnosis and classification of diabetes mellitus, provisional report of a WHO consultation. Diabetic Medicine 199815 539-553.

10 Kharitonenkov A, Wroblewski VJ, Koester A, Chen YF, Clutinger CK, Tigno XT, Hansen BC, Shanafelt AB \& Etgen GJ. The metabolic state of diabetic monkeys is regulated by fibroblast growth factor-21. Endocrinology $2007 \mathbf{1 4 8 7} 74-81$.

11 Garvey WT, Maianu L, Huecksteadt TP, Birnbaum MJ, Molina JM \& Ciaraldi TP. Pretranslational suppression of a glucose transporter protein causes insulin resistance in adipocytes from patients with non-insulin-dependent diabetes mellitus and obesity. Journal of Clinical Investigation 199187 1072-1081.

12 Chen WW, Li L, Yang GY, Li K, Qi XY, Zhu W, Tang Y, Liu H \& Boden G. Circulating FGF-21 levels in normal subjects and in newly diagnose patients with type 2 diabetes mellitus. Experimental and Clinical Endocrinology and Diabetes 2008116 65-68.

13 Li L, Yang G, Ning H, Yang M, Liu H \& Chen W. Plasma FGF-21 levels in type 2 diabetic patients with ketosis. Diabetes Research and Clinical Practice $2008 \mathbf{8 2} 209-213$.

14 Gälman C, Lundåsen T, Kharitonenkov A, Bina HA, Eriksson M, Hafström I, Dahlin M, Amark P, Angelin B \& Rudling M. The circulating metabolic regulator FGF21 is induced by prolonged fasting and PPAR $\alpha$ activation in man. Cell Metabolism $2008 \mathbf{8}$ 169-174.

15 Suzuki M, Uehara Y, Motomura-Matsuzaka K, Oki J, Koyama Y, Kimura M, Asada M, Komi-Kuramochi A, Oka S \& Imamura T. $\beta$ Klotho is required for FGF21 signaling through FGFR1c and FGFR3c. Molecular Endocrinology 200822 1006-1014.

16 Kharitonenkov A, Dunbar JD, Bina HA, Bright S, Moyers JS, Zhang C, Ding L, Micanovic R, Mehrbod SF, Knierman MD, Hale JE, Coskun T \& Shanafelt AB. FGF-21/FGF-21 receptor interaction and activation is determined by $\beta$ Klotho. Journal of Cellular Physiology 2008215 1-7.

Received 31 May 2009

Accepted 12 June 2009 